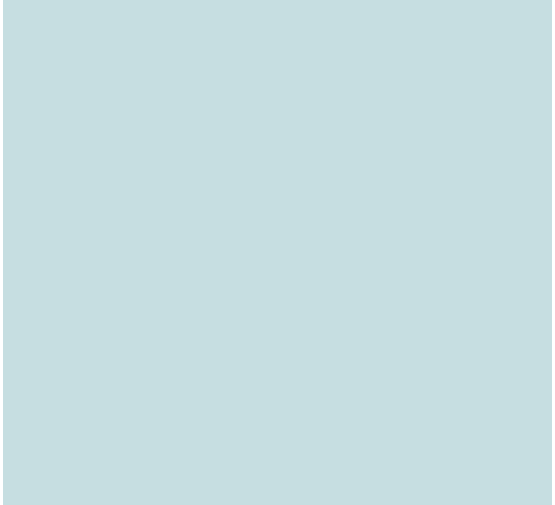

Breast Cancer: Assessing Response to Neoadjuvant Chemotherapy by Using US-guided Near-Infrared Tomography ${ }^{1}$

Quing Zhu, PhD

Patricia A. DeFusco, MD

Andrew Ricci, Jr, MD

Edward B. Cronin, MD

Poornima U. Hegde, MD

Mark Kane, MD

Behnoosh Tavakoli, MS

Yan Xu, MS

Jesse Hart, DO

Susan H. Tannenbaum, MD
1 From the Biomedical Engineering Program, Electrical and Computer Engineering Department, University of Connecticut, 371 Fairfield Rd, U2157, Storrs, CT 06269 (Q.Z., B.T., Y.X.); Departments of Pathology (A.R.) and Radiology (E.B.C.), Hartford Hospital, Hartford, Conn; and Department of Pathology (P.U.H.), Department of Radiology (M.K.), and Neag Cancer Center (S.H.T.), University of Connecticut Health Center, Farmington, Conn. Received November 18, 2011; revision requested January 16, 2012; revision received April 24; accepted May 7; final version accepted August 23. Supported by the Donaghue Medical Research Foundation. Address correspondence to Q.Z. (e-mail: zhu@engr.uconn.edu).
Purpose:

Materials and Methods:

Results:

Discussion:
To assess initial breast tumor hemoglobin $(\mathrm{Hb})$ content before the initiation of neoadjuvant chemotherapy, monitor the $\mathrm{Hb}$ changes at the end of each treatment cycle, and correlate these findings with tumor pathologic response.

The HIPAA-compliant study protocol was approved by the institutional review boards of both institutions. Written informed consent was obtained from all patients. Patients who were eligible for neoadjuvant chemotherapy were recruited between December 2007 and May 2011, and their tumor $\mathrm{Hb}$ content was assessed by using a near-infrared imager coupled with an ultrasonography (US) system. Thirty-two women (mean age, 48 years; range, 32-82 years) were imaged before treatment, at the end of every treatment cycle, and before definitive surgery. The patients were graded in terms of their final pathologic response on the basis of the Miller-Payne system as nonresponders and partial responders (grades 1-3) and near-complete and complete responders (grades 4 and $5)$. Tumor vascularity was assessed from total $\mathrm{Hb}(\mathrm{tHb})$, oxygenated $\mathrm{Hb}$ (oxyHb), and deoxygenated $\mathrm{Hb}$ (deoxyHb) concentrations. Tumor vascularity changes during treatment were assessed from percentage $\mathrm{tHb}$ normalized to the pretreatment level. A two-sample two-sided $t$ test was used to calculate the $P$ value and to evaluate statistical significance between groups. Bonferroni-Holm correction was applied to obtain the corrected $P$ value for multiple comparisons.

There were 20 Miller-Payne grade 1-3 tumors and 14 grade 4 or 5 tumors. Mean maximum pretreatment tHb, oxyHb, and deoxyHb levels were significantly higher in grade 4 and 5 tumors than in grade $1-3$ tumors $(P=.005$, $P=.008$, and $P=.017$, respectively). The mean percentage $\mathrm{tHb}$ changes were significantly higher in grade 4 or 5 tumors than in grade 1-3 tumors at the end of treatment cycles $1-3(P=.009$ and corrected $P=.009, P=.002$ and corrected $P=.004$, and $P<.001$ and corrected $P<.001$, respectively).

These findings indicate that initial tumor $\mathrm{Hb}$ content is a strong predictor of final pathologic response. Additionally, the $\mathrm{tHb}$ changes during early treatment cycles can further predict final pathologic response.

${ }^{\circ}$ RSNA, 2012

Supplemental material: http://radiology.rsna.org/lookup /suppl/doi:10.1148/radiol.12112415/-/DC1 
$\mathbf{P}$ reoperative or neoadjuvant chemotherapy is used in treating patients with locally advanced breast cancers as well as patients whose cancers are resectable but are not amenable to breast-conserving surgery (1-2). Histologic documentation of tumor response to preoperative chemotherapy is correlated with clinical outcome. An absence of residual tumor cells in the primary tumor bed after neoadjuvant therapy is strongly correlated with improved disease-free survival and overall survival (3). Surprisingly, neoadjuvant chemotherapy does not improve survival in the majority of patients who demonstrate lesser degrees of pathologic response. This is due in part to the standard "one treatment fits all" way clinical trials have been performed in the past. With the trend toward personalized treatment, accurate prediction of responses becomes more critical and may therefore improve survival.

\section{Advances in Knowledge}

- Pretreatment breast tumor hemoglobin $(\mathrm{Hb})$ content is a strong predictor of response to neoadjuvant chemotherapy: For the near-complete and complete responder group (Miller-Payne grades 4 and 5), the mean maximum total $\mathrm{Hb}(\mathrm{tHb})$ was 107.9 $\mu \mathrm{mol} / \mathrm{L} \pm 33.9$ (standard deviation) and the mean average $\mathrm{tHb}$ was $72.2 \mu \mathrm{mol} / \mathrm{L} \pm 24.5$, whereas for the nonresponder and partial responder group (grades 1-3), the mean maximum $\mathrm{tHb}$ was $75.7 \mu \mathrm{mol} / \mathrm{L} \pm$ 18.8 and the mean average $\mathrm{tHb}$ was $51.0 \mu \mathrm{mol} / \mathrm{L} \pm 13.9(P=$ .005 and $P=.009$, respectively).

- The percentage of $\mathrm{tHb}$ changes normalized to the pretreatment level can be used to predict pathologic tumor response at early treatment cycles; the differences between the two responder groups were statistically significant at the end of cycles 1-3 (corrected $P=.009$, corrected $P$ $=.004$, and corrected $P<.001$, respectively).
Several tumor markers are routinely used to predict treatment outcome and select therapy (4-6). Classifying breast cancers into subgroups on the basis of estrogen receptor (ER), progesterone receptor $(\mathrm{PR})$, and human epidermal growth factor receptor 2 (HER2/neu) status has improved our understanding of tumor response and has helped guide tailored treatment (7-8). However, these subgroup analyses are imperfect; within and among these subgroups, the response to chemotherapy varies widely. Furthermore, the group of "triple-negative” (ER/PR/HER2-negative) tumors does not, as yet, allow targeted therapy. Therefore, it is important to predict outcome and assess early tumor response, so treatment regimens can be appropriately tailored.

Conventional methods, including clinical examination, ultrasonography (US), and mammography have been shown to be moderately useful in the assessment of tumor response (9). Contrast material-enhanced magnetic resonance (MR) imaging has been used to assess breast cancers before treatment and prior to surgery for treatment and surgical planning (10-11). Fluorine 18 fluorodeoxyglucose positron emission tomography (PET) has been shown to demonstrate early metabolic changes that may correlate with final pathologic response (12). However, both MR imaging and PET require the injection of contrast agents and are costly for repeated use during treatment.

In the past decade, optical tomography and spectroscopy with near-infrared (NIR) diffused light have demonstrated

\section{Implications for Patient Care}

- The near-infrared technique with US localization of breast tumors can potentially be used to manage neoadjuvant chemotherapy by helping predict pathologic response even before the initiation of treatment.

- Monitoring of tumor response during early treatment cycles further enhances its predictive utility, potentially allowing the personalization of chemotherapy regimens. great potential in the initial diagnosis of a tumor (13-22) and in the assessment of the tumor vasculature's response to neoadjuvant chemotherapy (23-28). The NIR technique uses the intrinsic contrast of hemoglobin $(\mathrm{Hb})$, which is directly related to tumor angiogenesis development, a key process required for tumor growth and metastasis. Cost effectiveness, portability, lack of ionizing radiation, and the lack of need for contrast agents make NIR systems ideal for repeated use in clinical settings. Tomographic imaging with a pure NIR light technique is challenging because of poor lesion localization owing to light scattering. We have developed a US-guided NIR imaging technique that utilizes US to localize the light illumination and guide image reconstruction $(18,21,29-30)$. In a pilot study, we demonstrated the feasibility of using this technique to monitor tumor $\mathrm{Hb}$ changes during neoadjuvant

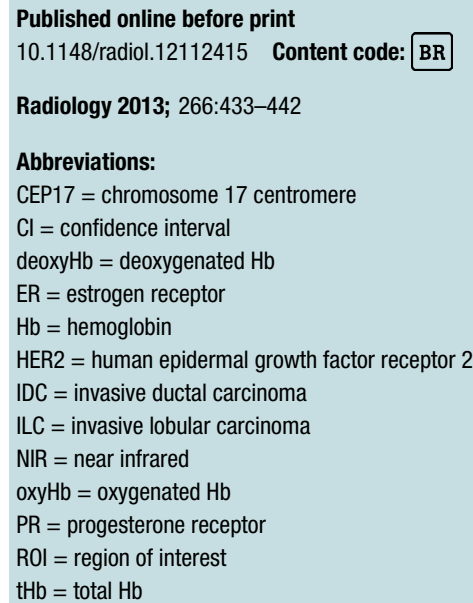

Author contributions:

Guarantors of integrity of entire study, Q.Z., E.B.C.; study concepts/study design or data acquisition or data analysis/ interpretation, all authors; manuscript drafting or manuscript revision for important intellectual content, all authors: manuscript final version approval, all authors; literature research, Q.Z., A.R., E.B.C., P.U.H., S.H.T.; clinical studies, Q.Z., P.A.D., E.B.C., P.U.H., M.K., B.T., Y.X., J.H., S.H.T.; experimental studies, Q.Z., A.R., E.B.C., B.T., Y.X.; statistical analysis, Q.Z.; and manuscript editing, Q.Z., P.A.D., A.R., E.B.C., P.U.H., M.K., S.H.T.

\section{Funding:}

This research was supported by the National Institutes of Health (grant R01EB002136).

Conflicts of interest are listed at the end of this article. 
chemotherapy (25). The purpose of this study was to evaluate initial tumor $\mathrm{Hb}$ content and early $\mathrm{Hb}$ changes during neoadjuvant chemotherapy as predictors of final tumor pathologic response with a larger patient pool.

\section{Materials and Methods}

\section{Patients}

The study protocol was approved by the institutional review boards of both institutions and was compliant with the terms of the Health Insurance Portability and Accountability Act. Written informed consent was obtained from all patients. Forty patients who were referred for neoadjuvant chemotherapy to two medical oncologists (P.A.D. and S.H.T.) were recruited from December 2007 to May 2011 at two hospitals. Two patients did not complete the study, as a result of a change in their treatment plan or a desire to withdraw from the study. Three patients underwent their first NIR US examination several days after the initiation of chemotherapy because of scheduling problems. The other 35 patients were imaged with NIR US prior to initiation of chemotherapy, at the end of every treatment cycle during chemotherapy, and prior to definitive surgery. Of these 35 patients, three were excluded from data analysis: One had inflammatory breast cancer with no measurable vascular content, another was an elderly patient with an HER2positive tumor who was treated with weekly sequential chemotherapy for 6 weeks longer than the remainder of the patients in the HER2-positive group, and the third patient had undergone core biopsy 3 days before pretreatment NIR US measurements.

Among the 32 patients from whom data were collected and analyzed (Table 1), 20 were treated with dose-dense doxorubicin, cyclophosphamide, and paclitaxel. This cohort was monitored at the end of each 2-week treatment cycle. The following groups of patients were monitored at the end of each 3-week treatment cycle: Patients treated with docetaxel and cyclophosphamide $(n=$ 4); patients treated with docetaxel and carboplatin with trastuzumab (HER2 positive, $n=5$ ); and patients treated according to the National Surgical Adjuvant Breast and Bowel Project B4-0 trial protocol, who received doxorubicin, cyclophosphamide, docetaxel, and bevacizumab (an antiangiogenic agent), with $(n=2)$ or without $(n=1)$ capecitabine.

Among the 32 patients (mean age, 48 years; range, 32-82 years), six underwent the first NIR US study before core biopsy, while 26 patients were imaged after core biopsy, with an average interval of 22 days (median, 22 days; range, 9-49 days). The first cycle of neoadjuvant chemotherapy was after the initial NIR US study (median, 0 days; range, $0-44$ days). The average interval between posttreatment NIR US and surgery was 8 days (median, 8 days; range, 0-35 days). During treatment, the NIR US studies were performed on the same day before scheduled chemotherapy unless scheduling or weather problems interfered (median, 0 days; range, 0-4 days). Among the 32 patients, 16 had a metal clip inserted before chemotherapy to mark the site of biopsy and to guide subsequent US imaging and surgical resection. Among the 32 patients, 26 underwent both preand posttreatment MR imaging. Four patients underwent pretreatment MR imaging but not posttreatment MR imaging, either because they underwent early surgery after clinical evidence showed disease progression or because they decided on mastectomy. One patient transferred from another hospital after core biopsy did not undergo pretreatment MR imaging, and one patient did not undergo MR imaging at all because of claustrophobia. The median interval between the pretreatment MR imaging examination and the first treatment was 17 days (range, 1-41 days); the median interval between the posttreatment MR imaging examination and surgery was 21 days (range, 3-49 days).

The histologic type of cancer was IDC in 21 patients; ILC in five patients; and invasive mammary carcinoma with mixed ductal and lobular features, or IDC/ILC, in six patients. Two patients had two distinct tumor masses in the same breast with similar characteristics.
Invasive carcinoma within the pretreatment core biopsies was graded by using the Nottingham histologic score. Testing for ER, PR, and HER2/neu immunohistochemistry was performed on formalin-fixed, paraffin-embedded core biopsy tissue. ER and PR statuses were scored by using the modified San Antonio scoring system, where total scores range from 0 to 8 and scores of $0-2$ are considered negative, a score of 3 is equivocal, and scores of 4 or greater are positive. Testing for HER2/neu gene amplification was performed by using the fluorescence in situ hybridization technique. The results were reported as the ratio of HER2/neu to CEP17. A ratio greater than 2.2 is considered positive for amplification of this gene, a ratio of less than 1.8 is considered negative, and a ratio between 1.8 and 2.2 is scored as equivocal. All assays were performed on pretreatment core biopsy samples.

\section{Pathologic Response Assessment}

Pathologic response was assessed by applying the Miller-Payne grading criteria to definitive surgery specimens in comparison with initial core biopsy samples. Two breast pathologists (A.R., with 27 years of experience, and P.U.H., with 10 years of experience) individually evaluated most cases in their respective hospitals. In three complex cases, hematoxylin-eosin-stained slides were reviewed by both pathologists in consultation, and a consensus was reached.

In the Miller-Payne system (31), pathologic response is divided into five grades on the basis of comparison of tumor cellularity between pre-neoadjuvant therapy core biopsy specimens and definitive surgical specimens as follows: A grade of 1 indicated no change or some alteration in individual malignant cells but no reduction in overall cellularity (pathologic nonresponse); a grade of 2 , minor loss of tumor cells but still high overall cellularity, of up to $30 \%$ (pathologic partial response); a grade of 3 , an estimated reduction in tumor cells of between $30 \%$ and 90\% (pathologic partial response); a grade of 4 , a marked disappearance 


\section{Table 1}

\section{Patient Demographic, Tumor, and Treatment Data}

\begin{tabular}{|c|c|c|c|c|c|c|c|c|}
\hline $\begin{array}{l}\text { Patient } \\
\text { Age (y) }\end{array}$ & $\begin{array}{l}\text { Tumor } \\
\text { Type }\end{array}$ & $\begin{array}{l}\text { Nottingham } \\
\text { Score (out of 9) }\end{array}$ & $\begin{array}{l}\text { Mitotic Count } \\
\text { (per } 10 \mathrm{HPF} \text { ) }\end{array}$ & $\begin{array}{l}\text { Receptor } \\
\text { Positivity }\end{array}$ & $\begin{array}{l}\text { Treatment } \\
\text { Regimen(s) }\end{array}$ & $\begin{array}{l}\text { Initial Tumor Size Measured at } \\
\text { US/MR Imaging (cm) }\end{array}$ & $\begin{array}{l}\text { Gross Residual } \\
\text { Tumor Size (cm) }\end{array}$ & $\begin{array}{l}\text { Miller-Payne } \\
\text { Grade }\end{array}$ \\
\hline 32 & IDC/ILC & 9 & 20 & $\mathrm{ER}+/ \mathrm{PR}+/ \mathrm{HER} 2-$ & ACT & $3.0 \times 1.9 / 3.8 \times 3.5 \times 5.6$ & 2.5 & 2 \\
\hline 51 & IDC & 8 & 8 & $\mathrm{ER}+/ \mathrm{PR}+/ \mathrm{HER} 2-$ & ACT & $3.0 \times 1.5 / 2.2 \times 2.2 \times 4.4$ & 2.3 & 2 \\
\hline 42 & IDC & 8 & 8 & ER-/PR-/HER2- & ACT & $2.3 \times 1.8 / 2.0 \times 3.3 \times 5.1$ & 0.0 (DCIS) & 5 \\
\hline $64^{*}$ & IDC & 9 & 34 & ER-/PR-/HER2e ${ }^{\dagger}$ & ACT & $2.8 \times 2.0 / 2.3 \times 4.2 \times 2.9$ & 0.0 (DCIS) & 5 \\
\hline 48 & IDC & 4 & 0 & ER-/PR-/HER2- & ACT & $3.6 \times 2.7 / 3.6 \times 3.3 \times 6.5$ & 0.7 & 3 \\
\hline 34 & IDC & 6 & 2 & $\mathrm{ER}+/ \mathrm{PR}+/ \mathrm{HER} 2-$ & ACT & $4.0 \times 2.0^{\ddagger} / 6.1 \times 3.9 \times 5.6$ & $>5.0$ & 1 \\
\hline 39 & ILC & 7 & 1 & $\mathrm{ER}+/ \mathrm{PR}+/ \mathrm{HER} 2-$ & ACT & $3.9 \times 1.3^{\ddagger} / 3.3 \times 2.4 \times 6.4$ & 1.9 & 2 \\
\hline 48 & IDC/ILC & 5 & 6 & $\mathrm{ER}+/ \mathrm{PR}+/ \mathrm{HER} 2-$ & ACT & $2.7 \times 2.3^{\ddagger} / 6.4 \times 6.9 \times 1.1$ & 5.4 & 1 \\
\hline 39 & IDC & 9 & 14 & ER-/PR-/HER2- & ACT & $3.6 \times 1.8^{\ddagger} / 4.2 \times 5.2 \times 9.7$ & 6.1 & 1 \\
\hline 42 & ILC & 3 & 0 & $\mathrm{ER}+/ \mathrm{PR}+/ \mathrm{HER} 2-$ & ACT & $2.8 \times 1.8^{\ddagger} / 7.6 \times 7.9 \times 6.4$ & 1.0 & 1 \\
\hline 38 & IDC & 9 & 20 & ER-/PR-/HER2- & ACT & $4.8 \times 2.2 / 2.5 \times 4.6 \times 5.4$ & 0.0 & 5 \\
\hline 49 & IDC & 6 & 8 & $\begin{array}{l}\mathrm{ER}+/ \mathrm{PR}+/ \mathrm{HER}^{+} \mathrm{e}^{\dagger} \\
\text { (FISH-) }\end{array}$ & ACT & $3.8 \times 2.7^{\ddagger} / 5.9 \times 5.1 \times 3.9$ & 7.5 & 3 \\
\hline $63^{\S}$ & IDC & 8 & 10 & $\begin{array}{l}\mathrm{ER}+/ \mathrm{PR}+/ \mathrm{HER}^{+} \mathrm{e}^{\dagger} \\
(\mathrm{FISH}-)\end{array}$ & ACT & $\begin{array}{l}3.4 \times 1.9 / 2.5 \times 1.4 / \mathrm{MR} \\
\text { imaging: } \mathrm{NA}\end{array}$ & 9.5 & 3 \\
\hline 37 & IDC/ILC & 7 & 8 & ER+/PR+/HER2- & ACT & $2.3 \times 1.3 / 5.0 \times 3.0 \times 4.3$ & 1.2 & 4 \\
\hline 35 & IDC & 9 & 39 & ER+/PR-/HER2- & ACT & $2.5 \times 1.7 / 2.3 \times 2.5 \times 2.0$ & 0.3 & 4 \\
\hline 40 & IDC & 4 & $1-3$ & $\mathrm{ER}+/ \mathrm{PR}+/ \mathrm{HER} 2-$ & ACT & $2.6 \times 1.6 / 3.1 \times 2.1 \times 1.7$ & 2.7 & 2 \\
\hline 44 & IDC & 6 & 5 & ER+/PR+/HER2- & ACT & $3.2 \times 1.3 / 3.0 \times 2.6 \times 1.5$ & 2.0 & 3 \\
\hline 53 & IDC & 9 & 58 & ER-/PR-/HER2- & ACT & $2.4 \times 1.9 / 3.1 \times 4.2 \times 2.3$ & 0.0 & 5 \\
\hline 47 & IDC & 9 & 15 & ER+/PR-/HER2- & ACT & $3.6 \times 3.2 / 4.2 \times 4.7 \times 5.5$ & 0.0 & 5 \\
\hline 53 & ILC & 6 & $0-1$ & $\mathrm{ER}+/ \mathrm{PR}+/ \mathrm{HER} 2-$ & ACT & $1.9 \times 1.4 / 2.2 \times 1.8 \times 1.3$ & 5.0 & 2 \\
\hline 32 & IDC & 9 & 16 & ER-/PR-/HER2+ & $\mathrm{TCH}$ & $2.0 \times 1.3 / 1.3 \times 1.8 \times 2.3$ & 0.2 & 4 \\
\hline 64 & IDC/ILC & 6 & 4 & $\begin{array}{l}\text { ER-/PRe/HER2e }{ }^{\dagger} \\
(\text { FISH+) }\end{array}$ & $\mathrm{TCH}$ & $6.5 \times 2.0 / 3.2 \times 3.2 \times 5.4$ & 2.0 & 4 \\
\hline 55 & IDC/ILC & 7 & 10 & ER+/PR-/HER2+ & $\mathrm{TCH}$ & $2.9 \times 2.0 / 3.2 \times 4.8 \times 5.2$ & 0.0 & 5 \\
\hline 40 & IDC & 9 & 30 & ER+/PR+/HER2+ & $\mathrm{TCH}$ & $3.3 \times 1.0 / 1.7 \times 1.2 \times 1.8$ & 1.3 & 3 \\
\hline 38 & IDC & 7 & 9 & $\begin{array}{l}\mathrm{ER}+/ \mathrm{PR}+/ \mathrm{HER} 2- \\
(\mathrm{FISH}+)\end{array}$ & $\mathrm{TCH}$ & $1.7 \times 1.0^{\ddagger} / 2.8 \times 1.4 \times 2.4$ & 0.4 (DCIS) & 4 \\
\hline 64 & IDC/LC & 8 & 44 & ER+/PR-/HER2- & TC & $5.2 \times 3.0 / 3.9 \times 5.5 \times 4.3$ & & 2 \\
\hline 48 & ILC & 6 & $0-2$ & ER+/PR-/HER2- & TC & $5.0 \times 1.5^{\ddagger} / 5.1 \times 5.4 \times 3.4$ & 5.8 & 2 \\
\hline 69 & IDC & 7 & 16 & ER+/PR-/HER2- & TC & $2.8 \times 1.7^{\ddagger} / 2.8 \times 4.4 \times 6.6$ & 2.1 & 3 \\
\hline 82 & IDC & 6 & 0 & ER+/PR-/HER2- & TC & $5.2 \times 2.2^{\ddagger} / \mathrm{MR}$ imaging: NA & 10.0 & 1 \\
\hline $47^{\S}$ & IDC & 8 & 10 & ER+/PR-/HER2- & ACT plus bev & $\begin{array}{l}6.4 \times 1.7^{\ddagger} / 4.0 \times 2.0^{\ddagger} / 5.4 \times \\
\quad 5.4 \times 10.2^{\| \prime}\end{array}$ & 0.0 (DCIS) & 5 \\
\hline 54 & IDC & 9 & 26 & ER-/PR+/HER2- & ACT plus bev & $2.3 \times 1.4 / 2.7 \times 3.1 \times 2.2$ & 0.0 & 5 \\
\hline 42 & ILC & 6 & 5 & ER+PR+HER2- & ACT plus bev & $3.9 \times 1.5 / 3.7 \times 4.4 \times 4.0$ & 6.5 & 2 \\
\hline
\end{tabular}

Note.-ACT = doxorubicin, cyclophosphamide, and paclitaxel; bev = bevacizumab; DCIS = ductal carcinoma in situ; FISH = fluorescence in situ hybridization; HPF = high-power field; IDC = invasive ductal carcinoma; ILC = invasive lobular carcinoma; NA = not applicable; TC = docetaxel and cyclophosphamide; TCH = docetaxel and carboplatin with trastuzumab. Initial tumor size was measured in the $\mathrm{x}$ - and $\mathrm{z}$-axis dimensions at US and in the craniocaudal, transverse, and anteroposterior dimensions at MR imaging.

* This patient had clear evidence of tumor heterogeneity, with the vast majority of cells being HER2 negative at immunohistochemistry. A discrete clone of cells was HER2 equivocal at immunohistochemistry. This minor clone was HER2 positive at fluorescence in situ hybridization (HER2-chromosome 17 centromere [CEP17] ratio = 4.4); however, the remainder of the tumor was negative for HER2 at fluorescence in situ hybridization.

${ }^{\dagger}$ HER2 equivocal.

‡ Patients had initially palpable tumors with ill-defined and heterogeneous US images. Tumor size was an estimate.

$\S$ This patient had two distinct tumors in the same breast with the same characteristics.

" US images showed two distinct tumors with ill-defined margins located at the 11- and 1-0'clock positions, and MR imaging showed one tumor mass.

of tumor cells such that only small clusters or widely dispersed individual cells remained, with more than $90 \%$ loss of tumor cells (almost pathologic complete response); and a grade of 5 , no malignant cells identifiable in slices from the site of the tumor and only vascular fibroelastic stroma remaining, often containing macrophages (however, ductal carcinoma in situ may be present) (pathologic complete response). 


\section{US and NIR System and Imaging}

US examinations were performed with either an IU22 unit with an L12 linear transducer (Philips Medical Systems, Bothell, Wash) or a Sequoia unit with a $15 \mathrm{~L} 8$ linear transducer (Acuson, Mountain View, Calif). Two NIR systems with identical designs were used at the two hospitals, and details about these systems have been given previously (32). Briefly, the probe consists of the commercial US transducer located in the middle, with source and detector light guides (optical fibers) distributed at the periphery. Four laser diodes with 740-, 780-, 808-, and 830$\mathrm{nm}$ optical wavelengths were sequentially switched to nine positions on the probe, while the reflected light was coupled by the light guides to 10 parallel detectors. The entire NIR data acquisition interval was less than 5 seconds. For each patient, US images and optical measurements were acquired simultaneously before treatment at multiple locations, including the lesion region and a normal region of the contralateral breast in the same quadrant as the lesion. The optical data acquired in the normal contralateral breast were used as a reference for calculating the background optical absorption and reduced scattering coefficients that were used in the image reconstruction of the lesions. The US and optical measurements were repeated at the end of each treatment cycle and before the surgery.

Details of the optical imaging reconstruction algorithm with experimental validation have been described elsewhere (33). Briefly, the NIR reconstruction takes advantages of US localization of lesions and segments the imaging volume into a region of interest (ROI) and background nonlesion regions. Because the spatial resolution of diffused light is poorer than that of US, the ROI is chosen to be at least two to three times larger than that seen by using US in the $\mathrm{x}$ - and $\mathrm{y}$-axis dimensions. In this study, because of the large tumor size, the ROI in the $\mathrm{x}^{-}$and $\mathrm{y}$-axis dimensions was chosen to be $10 \mathrm{~cm}$, which is the size of the probe in most cases. In addition, because the depth localization of diffused light is very poor, a tighter ROI in the depth dimension was set by using coregistered US. For each patient, the same size ROI obtained from the pretreatment US examination was used for processing all data sets obtained at different treatment cycles. Therefore, the changes in tumor size seen with US during treatment had no major effect on NIR image reconstruction. Among the 32 patients, 11 had initially palpable tumors with ill-defined and heterogeneous pretreatment US images. For these patients, tumor sizes estimated from pretreatment MR images were used to assist the determination of the US ROI in the $\mathrm{x}$ - and $\mathrm{y}$-axis dimensions. The ROI in the depth dimension was typically set from the top border of the ill-defined tissue pattern to the chest wall as seen with US.

The optical absorption distribution at each wavelength was reconstructed, and total $\mathrm{Hb}(\mathrm{tHb})$ concentration, as well as oxygenated $\mathrm{Hb}$ (oxyHb) concentration and deoxygenated $\mathrm{Hb}$ (deoxyHb) concentration, was computed from absorption maps at the four wavelengths (34). Maximum and average $\mathrm{tHb}$, oxyHb, and deoxyHb were measured, and the average was computed within the volumetric zone exceeding $50 \%$ of the maximum value. For each patient imaged at each cycle, average values of maximum and average that were obtained from several quality NIR images at the tumor location were used to characterize the tumor. Data with patient motion as evaluated by using two coregistered US images before and after each NIR measurement were excluded from averaging. To assess each patient's response, the $\mathrm{tHb}$ obtained before treatment was taken as the baseline, and the percentage $\mathrm{tHb}$ normalized to the baseline was used to quantitatively evaluate the tumor vascular changes during chemotherapy. One author (Q.Z., with 12 years of experience in US and optical imaging) performed the optical imaging.

\section{MR Imaging, US, and Measurements}

MR images were obtained by using an MR imaging unit (Vista 1.5 T or Avanto 1.5 T, Siemens, Erlangen, Germany; Excite 1.5 T, GE Healthcare, Waukesha,
Wis) with three-dimensional flash $\mathrm{dy}$ namic acquisition with subtraction. Pre- and posttreatment images were available in 26 patients. The size of the tumor was measured on pre- and posttreatment images in the craniocaudal, transverse, and anteroposterior dimensions by two radiologists (E.B.C., with 23 years of experience, and M.K., with 13 years of experience) for cases from their respective hospital. The percentage ratio of the largest dimension of posttreatment measurements to the largest dimension of pretreatment measurements was used to compute the percentage reduction.

Twenty-one patients had well-defined tumors at US, and their tumor sizes were measured by US technologists in consultation with attending radiologists. The percentage ratio of the largest dimension of each posttreatment measurement to the largest dimension of pretreatment measurement was used to compute the percentage reduction in tumor size, which was noted as percentage reduction at US. For the 11 patients with initially palpable lesions but ill-defined and heterogeneous US images, pretreatment tumor sizes were estimated at the time of imaging. The tumor location at each treatment cycle was tracked by using previous US images as references. The tumor clock position, distance of the tumor to the nipple, and depth were documented for each case. Additionally, the tumor posterior shadowing, color Doppler profile, surrounding tissue structures, and metal clip position were also reviewed and used to help identify the tumor for each subsequent measurement. Seven of 20 Miller-Payne grade 1-3 tumors and nine of 14 grade 4 or 5 tumors had metal clips marking the biopsy sites.

\section{Statistical Analysis}

A two-sample two-sided $t$ test was used to calculate statistical significance for comparisons between groups, with $P$ $<.05$ considered to indicate a statistically significant difference. When comparing percentage $\mathrm{tHb}$ and percentage reduction at US at different treatment cycles, the Bonferroni-Holm correction was applied to obtain the corrected $P$ 


\section{Table 2}

\section{Pearson Correlation Coefficients and $\boldsymbol{P}$ Values between Miller-Payne Grades and Pretreatment Parameters}

\begin{tabular}{|c|c|c|c|c|c|c|c|}
\hline Statistic & Maximum/Average $\mathrm{tHb}$ & $\begin{array}{l}\text { Maximum/Average } \\
\mathrm{OxyHb}\end{array}$ & $\begin{array}{l}\text { Maximum/Average } \\
\text { DeoxyHb }\end{array}$ & $\begin{array}{l}\text { Nottingham } \\
\text { Score }\end{array}$ & $\begin{array}{l}\text { Mitotic Count (per } 10 \\
\text { High-Power Fields) }\end{array}$ & $\begin{array}{l}\text { Largest Tumor Dimension } \\
\text { at MR Imaging* }\end{array}$ & $\begin{array}{l}\text { Largest Tumor } \\
\text { Dimension at US }\end{array}$ \\
\hline $\begin{array}{l}\text { Correlation } \\
\text { coefficient }\end{array}$ & $0.531 / 0.497$ & $0.456 / 0.436$ & $0.538 / 0.523$ & 0.531 & 0.407 & 0.111 & 0.174 \\
\hline$P$ value & $.001 / .003$ & $.007 / .010$ & $.001 / .001$ & .001 & .017 & .552 & .417 \\
\hline
\end{tabular}

* In 30 patients with available pretreatment MR images.

† In 21 patients with 22 tumors with well-defined margins on pretreatment US images.

value for the number of treatment cycles. Bonferroni-Holm correction ranks all $P$ values from the smallest to the largest and evaluates statistical significance on the basis of $P(i)<\alpha /(n-i$ $+1)$ or $P_{c}(i)=(n-i+1) \cdot P(i)<\alpha$, where $\alpha$ is $.05, n$ is the total number of comparisons, $i$ starts from 1 (corresponding to the smallest $P$ value) to $n$ (corresponding to the largest $P$ value), and $P$ is the corrected $P$ value. Both the prediction accuracy and the specificity based on pretreatment maximum $\mathrm{tHb}$ were determined by selecting an optimal threshold by using the receiver operating characteristic curve that provided the best compromise between sensitivity and specificity. Pearson correlation was performed between each tumor's Miller-Payne grade and the pretreatment maximum and average tHb, oxyHb, and deoxyHb; tumor Nottingham score and mitotic counts assessed at core biopsy; and pretreatment MR and US imaging findings to compare the predictive value of each parameter with pathologic response. Software (Minitab 15; Minitab, State College, Pa) was used for statistical calculations.

\section{Results}

There were 20 grade $1-3$ tumors and 14 grade 4 or 5 tumors. For grade 4 or 5 tumors, the mean maximum $\mathrm{tHb}$ was $107.9 \mu \mathrm{mol} / \mathrm{L} \pm 33.9$ (standard deviation), and the mean average $\mathrm{tHb}$ was $72.2 \mu \mathrm{mol} / \mathrm{L} \pm 24.5$, whereas for grade 1-3 tumors, the mean maximum $\mathrm{tHb}$ was $75.7 \mu \mathrm{mol} / \mathrm{L} \pm 18.8$, and the mean average $\mathrm{tHb}$ was $51.0 \mu \mathrm{mol} / \mathrm{L} \pm 13.9(P$ $=.005$ and $P=.009$, respectively). The

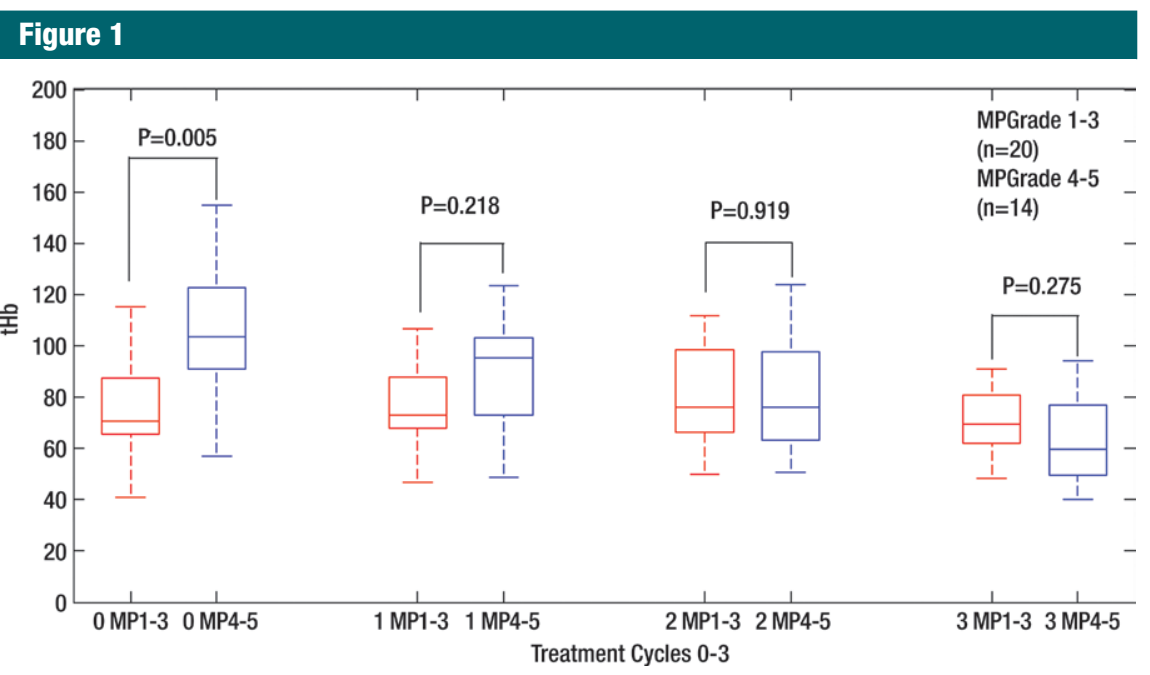

Figure 1: Box-and-whisker plot shows mean maximum tHb in micromoles per liter in Miller-Payne (MP) grade 1-3 tumors and grade 4 or 5 tumors before treatment (cycle 0 ) and at the ends of chemotherapy cycles 1-3.

mean differences in maximum and average $\mathrm{tHb}$ were $32.2 \mu \mathrm{mol} / \mathrm{L}(95 \%$ confidence interval [CI]: $11.2,53.2 \mu \mathrm{mol} / \mathrm{L})$ and $21.2 \mu \mathrm{mol} / \mathrm{L}$ (95\% CI: 5.9, 36.4 $\mu \mathrm{mol} / \mathrm{L})$, respectively. However, there was no significant difference at the end of treatment cycles 1-3 (Fig 1).

Before treatment, oxyHb and deoxy$\mathrm{Hb}$ were significantly different between grade $1-3$ and grade 4 or 5 tumors $(P$ $=.008$ and $P=.017$, respectively), but this was not true in subsequent measurements (Table E1 [online]). When we compared the predictive value of pretreatment $\mathrm{tHb}$, oxyHb, and deoxyHb with the predictive value of Nottingham score and mitotic index at core biopsy, pretreatment maximum $\mathrm{tHb}$ and deoxyHb achieved the highest predictive value $(r=0.531$ and $r=0.538, P=.001)$, followed closely by tumor Nottingham score $(r=0.531, P=.001$, Table 2$)$. We found no correlation between pretreatment tumor size at MR imaging $(P=$ $.552)$ or US $(P=.417)$ and Miller-Payne grade (Table 2 ).

We also evaluated prediction accuracy and specificity on the basis of pretreatment maximum $\mathrm{tHb}$ levels in grade 1-3 and grade 4 or 5 tumors (Fig 2). For each group, three tumor types (IDC, IDC/ILC, and ILC) were grouped together. However, there were no ILC tumors in the MillerPayne grade 4 or 5 group. An optimal threshold between 89 and $91 \mu \mathrm{mol} / \mathrm{L}$ yielded a situation in which 11 of 14 grade 4 or 5 tumors were above the threshold and 16 of 20 grade $1-3$ tumors were below it. The prediction accuracy was $79 \%$, and the specificity was $80 \%$. The mean maximum $\mathrm{tHb}$ 


\section{Figure 2}

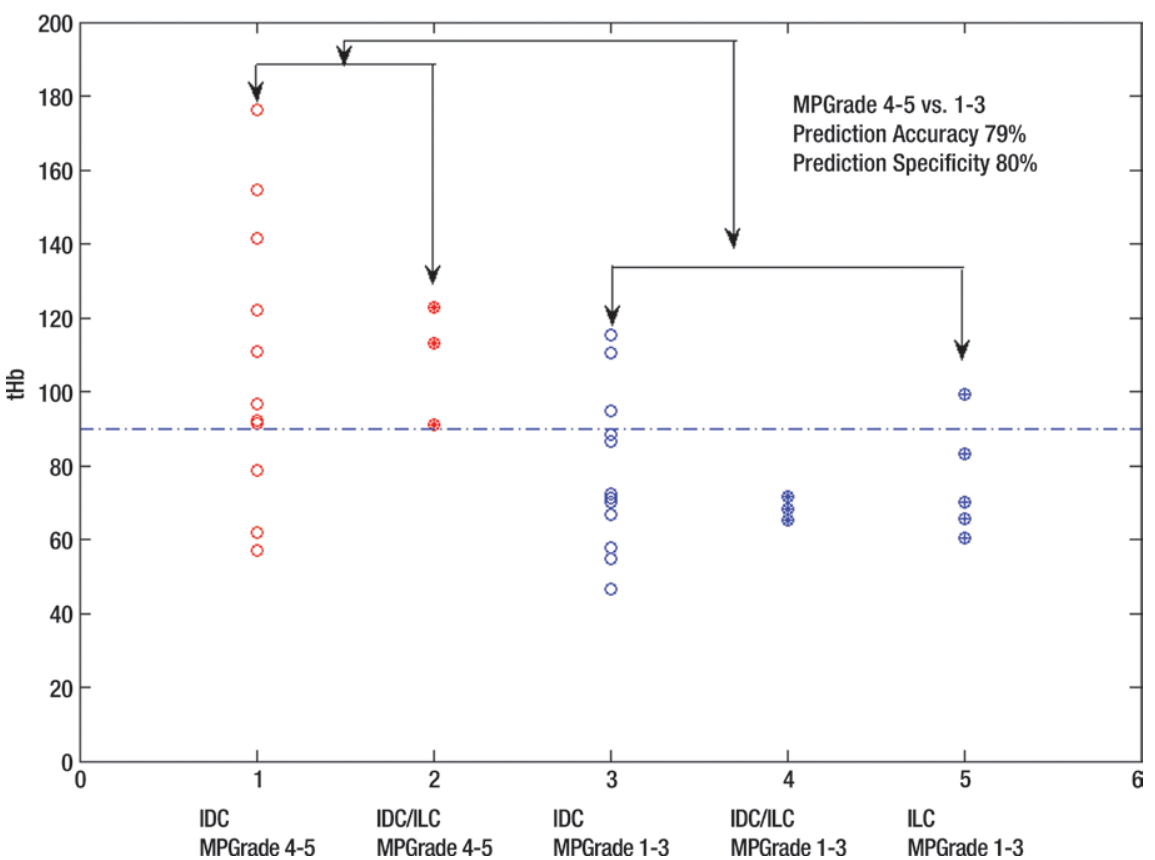

Figure 2: Scatterplot shows pretreatment maximum $\mathrm{tHb}$ in micromoles per liter for three types of tumors in two responder groups. $M P=$ Miller-Payne.

\section{Figure 3}

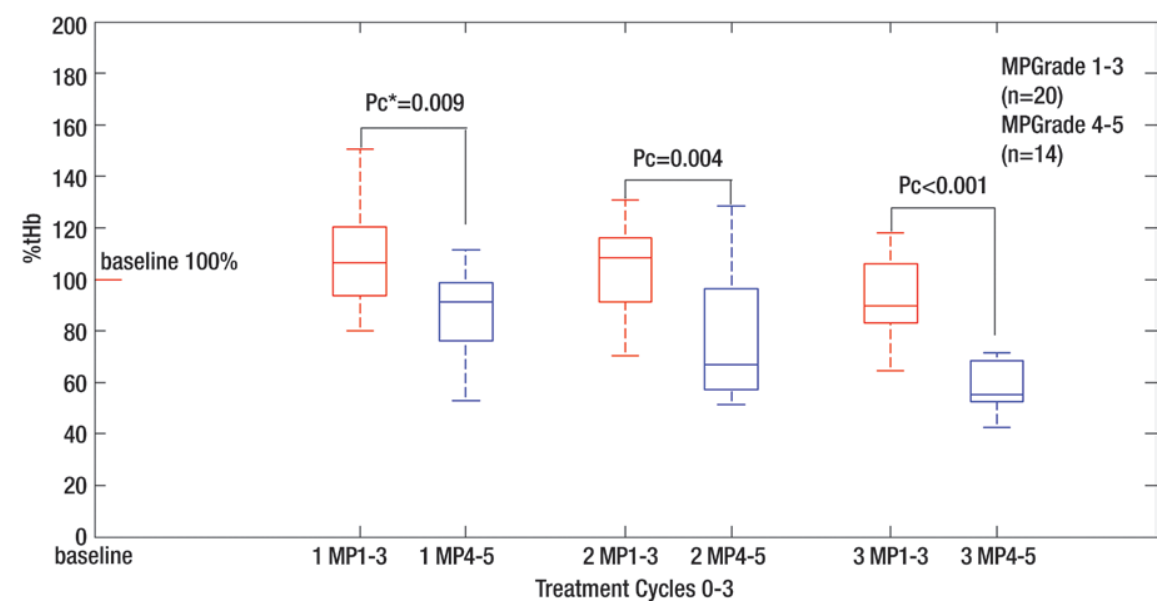

Figure 3: Box-and-whisker plot shows percentage $\mathrm{Hb}$ in grade $1-3$ and grade 4 or 5 tumors before treatment (cycle 0) and at the ends of chemotherapy cycles $1-3 . M P=$ Miller-Payne, $P C^{*}=$ Bonferroni-Holm corrected $P$ value (originally calculated with two-sample, two-sided $t$ test).

levels of grade $1-3$ tumors were similar for the three types of tumors (IDC: $77.5 \mu \mathrm{mol} / \mathrm{L} \pm 22.4$; IDC/ILC: 68.5 $\mu \mathrm{mol} / \mathrm{L} \pm 3.2$; and ILC: $75.7 \mu \mathrm{mol} / \mathrm{L}$ $\pm 15.6)$. The levels were significantly higher in grade 4 or 5 IDC and IDC/
Multiple NIR data sets were obtained at the tumor sites, and average $\mathrm{tHb}$ values were used to characterize each tumor. The mean standard deviations of pretreatment maximum $\mathrm{tHb}$ values of grade $1-3$ and grade 4 or 5 tumors were 5.9 and $4.9 \mu \mathrm{mol} / \mathrm{L}$, respectively. Thus, on average, $4.5 \%$ $(4.9 / 107.9)$ to $7.8 \%(5.9 / 75.7)$ changes could be encountered in repeated imaging by using the hand-held probe. The mean standard deviations of subsequent measurements were in a similar range-for example, the corresponding values were 5.6 and $5.9,6.1$ and 5.2 , and 5.2 and $6.1 \mu \mathrm{mol} / \mathrm{L}$ at the ends of cycles $1-3$ for grade $1-3$ and grade 4 or 5 tumors, respectively.

Percentage $\mathrm{tHb}$ based on maximum and average $\mathrm{tHb}$ was calculated, and the results for the two groups based on the maximum are given in Figure 3 and in Table E2 (online). Statistical significance was achieved at the end of cycle 1 . For grade 1-3 tumors, percentage $\mathrm{tHb}$ change was $109.8 \% \pm 27.7$, whereas for grade 4 or 5 tumors, percentage $\mathrm{tHb}$ change was $87.9 \% \pm 17.9$. The mean difference was $21.9 \%(P=.009$ and corrected $P=.009)$, and the $95 \%$ CI was $5.9 \%, 37.9 \%$. The significance remained high at the end of cycles 2 and $3(P=$ .002 and corrected $P=.004$ and $P<$ .001 and corrected $P<.001$, respectively). The percentage $\mathrm{tHb}$ changes in three patient groups in different treatment regimens are given in Appendix E1 (online). An example of complete pathologic response is shown in Figure 4, and examples of complete and partial pathologic response can be found in Figures E1-E4 (online).

The percentage changes in size at US of the largest dimension of grade 1-3 and grade 4 or 5 tumors were measured in 21 patients with 22 tumors that were well-defined at US (Table E2 [online]). For grade $1-3$ tumors $(n=11)$, percentage reduction in size at US was $82.7 \%$ $\pm 28.1,66.0 \% \pm 15.6$, and $59.2 \% \pm$ 13.0 at the end of cycles $1-3$, respectively; whereas for grade 4 or 5 tumors $(n=11)$, percentage reduction in size at US was $74.8 \% \pm 17.4,55.5 \% \pm 19.0$, $41.6 \% \pm 16.4$, respectively. The $P$ values were $.437, .172$, and .02 , respectively, 


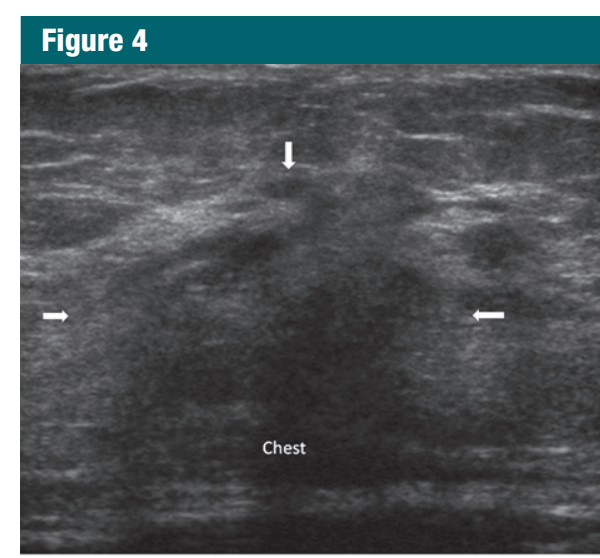

a.

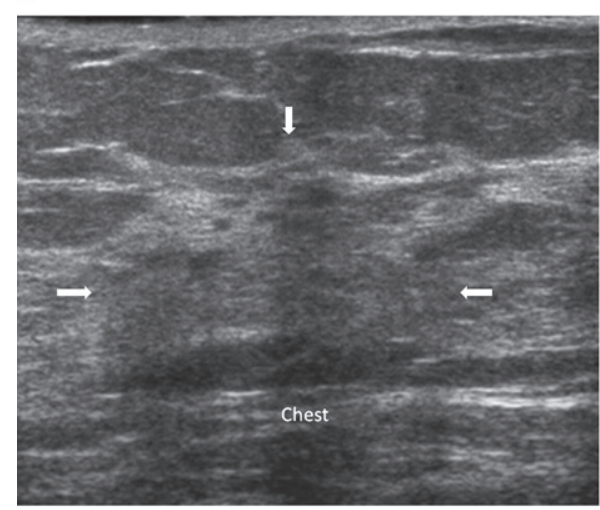

b.

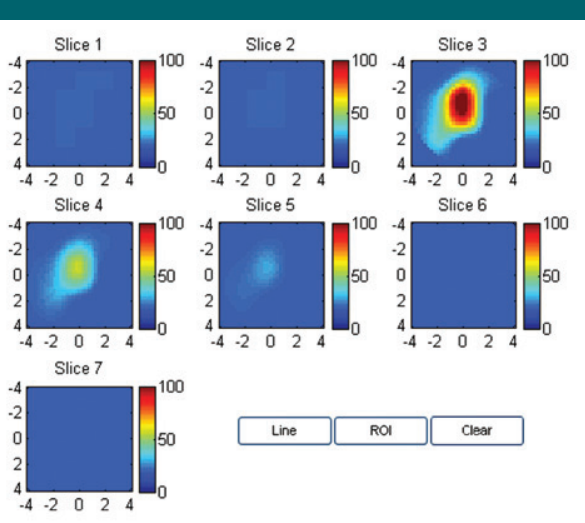

C.
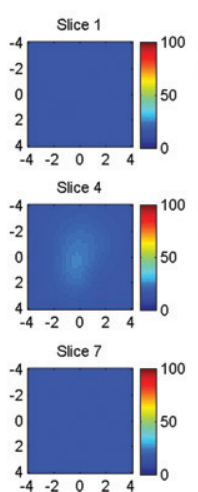

d.

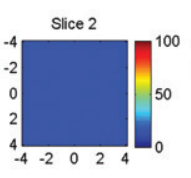

Slice 5
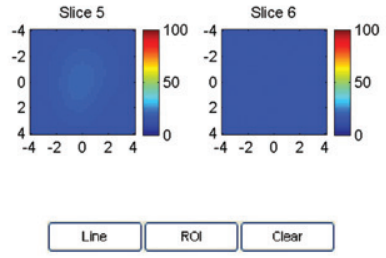

e.

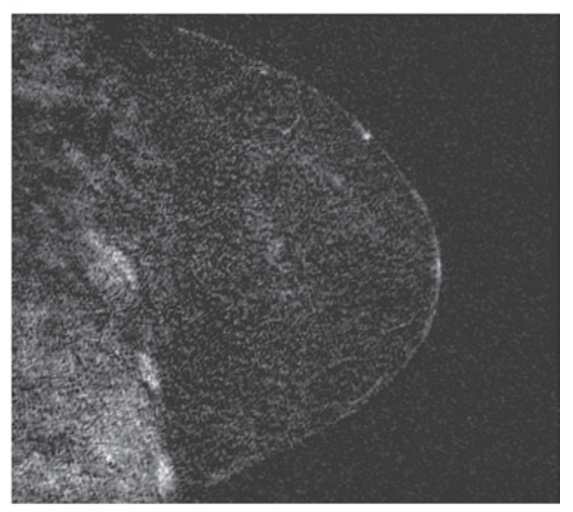

f.

Figure 4: Pathologic complete response in an HER2-positive tumor. Images in 55-year-old woman with intermediate-grade IDC treated with docetaxel and carboplatin with trastuzumab acquired $(\mathbf{a}, \mathbf{c}, \mathbf{e})$ before treatment and $(\mathbf{b}, \mathbf{d}, \mathbf{f})$ prior to surgery. $(\mathbf{a}, \mathbf{b})$ Sonograms show ill-defined heterogeneous tumor (arrows), which was palpable at the beginning of the treatment. (c, d) Maps of tHb concentration show spatial distribution of tHb concentration reconstructed from 0.5- to 3.5-cm depth range from the skin surface to the chest wall. Color bar $=\mathrm{tHb}$ in micromoles per liter. The tHb declined from $113 \mu \mathrm{mol} / \mathrm{L}$ before treatment to $33 \mu \mathrm{mol} / \mathrm{L}$ before surgery $(71 \%$ reduction). Dramatic reduction (of 55\%) occurred at the end of chemotherapy cycle 3 (Fig E1 [online]). (e) MR image obtained before treatment shows 5-cm tumor. (f) MR image obtained at end of treatment shows no visible residual tumor. This patient had a complete pathologic response with no residual tumor; her tumor was Miller-Payne grade 5.

and corrected $P$ values were $.0437, .344$, and .06 , respectively. At the end of cycle 3 , the difference between the two groups approached statistical significance.

The pretreatment MR imaging measurements of the largest dimension were $5.0 \mathrm{~cm} \pm 1.8$ and $4.7 \mathrm{~cm} \pm 2.2$, and the posttreatment measurements were $2.7 \mathrm{~cm} \pm 1.5$ and $0.7 \mathrm{~cm} \pm 1.1$, for grade 1-3 tumors and grade 4 or 5 tumors, respectively. The percentage reduction was $55.0 \% \pm 21.3$ for grade $1-3$ tumors and $21.1 \% \pm 28.2$ for grade 4 or 5 tumors $(P=.004)$.

\section{Discussion}

In the past 5 years, optical tomography and spectroscopy have been explored by several groups (23-28) for potential use in assessing chemotherapy response. A recent study (27) involving NIR spectroscopy measured percentage changes in oxyHb, deoxyHB, water, and optical scattering normalized to pretreatment values at 1,4 , and 8 weeks after neoadjuvant chemotherapy for 10 patients. All functional parameters differed significantly in responders and nonresponders at the 4-week examination, except for percentage water change. In our previous study (25), we imaged 11 patients by using the NIR US imager before treatment; after chemotherapy cycles 2,4 , and 6 ; and prior to surgery. We found a noticeable difference in $\mathrm{tHb}$ changes at the end of cycle 2 between responders and nonresponders. This study, with its larger patient pool, showed that percentage tHb over the first three treatment cycles was statistically different between Miller-Payne grade 4 or 5 tumors and grade $1-3$ tumors. Furthermore, the pretreatment maximum and mean average $\mathrm{tHb}$ levels were significantly higher in the tumors with the best pathologic response (grade 4 or 5) than in the tumors with modest or no response to chemotherapy (grades 1-3). This suggests that hypervascular tumors respond to standard cytotoxic, herceptin-based, and antiangiogenic therapies better and in a more substantive way than do hypovascular tumors, perhaps because of the better access of chemotherapeutic 
agents to the cancer cells. Given the relative hypovascularity of most ILCs, it is not surprising that there were no pathologic complete responses in this subgroup. Although US is useful in monitoring chemotherapy responses in patients with well-defined tumor margins, the early assessment of tumor size response is not quite possible at early treatment cycles $1-3$.

A closely related result was reported by Kuo et al (35), who assessed power Doppler US measurements obtained in 30 patients. They found that there was a tendency for response in tumors with relatively high initial vascularity. In a study (23) involving NIR spectroscopy, deoxyHb was found to decrease within the 1 st week of chemotherapy in responders. In another study, oxyHb was found to increase in responders on day 1 (28). We obtained the statistical significance of oxyHb and deoxyHb levels at pretreatment between the two responder groups. However, oxyHb and deoxyHb are not independent predictors, as their levels vary directly with the $\mathrm{tHb}$ level. Pearson correlation revealed that pretreatment $\mathrm{tHb}$ and deoxyHb have the highest predictive value for pathologic response. The results of our study and those of others cited have substantial implications for the use of NIR-measured tumor $\mathrm{Hb}$ content in predicting response to chemotherapy.

There were some limitations to this study. First, the majority of the patients were referred for neoadjuvant chemotherapy after core biopsy, and hence the baseline NIR US imaging examination was performed after the initial biopsy. A bruise or hematoma caused by prior biopsy could have had some effect on pretreatment NIR measurements. Second, patients treated with different regimens were pooled together to assess early response. Antiangiogenic therapy has a more profound effect on tumor vascular changes than standard cytotoxic and herceptin-based therapies. Future trials will control therapies and assess early tumor responses to specific regimens. Third, 16 of 32 patients had metal clips marking the biopsy sites, and their tumors could be readily tracked with US during chemotherapy.
Future trials will enforce the use of metal clips for all patients. Last, the maximum and average $\mathrm{Hb}$ levels over the image volume were used to quantify the initial tumor vascularity as well as the percentage changes from the initial values during treatment. Heterogeneous $\mathrm{Hb}$ distributions were observed in both responder groups on pretreatment images, and the distributions were more homogeneous toward the end of treatment. It is not clear that the heterogeneous distribution can be used to qualitatively characterize the two responder groups at pretreatment as well as during early treatment cycles on the basis of the limited data. Future trials will evaluate this important parameter.

The technical limitations of the USguided NIR technique include the accuracy of reconstructed optical absorption coefficients and the longitudinal repeatability of the measurements. For a large high-contrast phantom target of $5 \mathrm{~cm}$, about $55 \%-65 \%$ reconstruction accuracy on target absorption can be achieved (33). Because the average pretreatment tumor sizes of the two responder groups were similar, the underreconstruction should affect light quantification in both groups similarly. Therefore, the comparison of pretreatment and early treatment $\mathrm{Hb}$ levels between the two responder groups should be minimally affected. Additionally, because the same size ROI obtained in each patient at pretreatment was used for reconstructions at all subsequent treatment cycles, the underreconstruction should have had a minimal effect on the percentage $\mathrm{tHb}$, which was normalized to the pretreatment level. The longitudinal repeatability of reconstructed phantom absorption coefficient is about $5 \%-10 \%$. In a recent study (34), we validated the estimated oxygen saturation $\left(\mathrm{SO}_{2}\right)$, computed as $\mathrm{So}_{2}=$ oxyHb/ $\mathrm{tHb} \cdot 100$, by using a $\mathrm{Po}_{2}$ electrode as a reference, and the difference was less than $8 \%$ for blood phantoms of various oxygen conditions and sizes located at different depths.

In conclusion, our initial findings indicate that pretreatment tumor $\mathrm{Hb}$ content is a strong predictor of response to neoadjuvant chemotherapy and that percentage $\mathrm{tHb}$ changes normalized to the pretreatment level can be used to further identify responders and nonresponders at early treatment cycles. In the genomic era of personalized medicine, where monitoring of early responses for outcome prediction becomes crucial, our NIR technology could prove valuable.

Acknowledgments: Sandra Trifiro, RN, CCRC, Coordinator of Clinical Research Office of Hart ford Hospital, is specifically acknowledged for consenting and scheduling patients recruited at the Hartford Hospital. The staff of the Cancer Center of University of the Connecticut Health Center is thanked for their assistance in patient scheduling. The US technologists of the Radiology Department of the University of Connecticut Health Center and Jefferson $\mathrm{x}$-ray at Wethersfield were extremely helpful in assisting in US data acquisition and patent scanning.

Disclosures of Conflicts of Interest: Q.Z. Finan cial activities related to the present article: none to disclose. Financial activities not related to the present article: institution owns patents on technology development. Other relationships: none to disclose. P.A.D. No relevant conflicts of inter est to disclose. A.R. No relevant conflicts of interest to disclose. E.B.C. No relevant conflicts of interest to disclose. P.U.H. No relevant conflicts of interest to disclose. M.K. No relevant conflicts of interest to disclose. B.T. No relevant conflicts of interest to disclose. Y.X. No relevant conflicts of interest to disclose. J.H. No relevant conflicts of interest to disclose. S.H.T. No relevant conflicts of interest to disclose.

\section{References}

1. Fisher B, Brown A, Mamounas E, et al. Ef fect of preoperative chemotherapy on localregional disease in women with operable breast cancer: findings from National Surgical Adjuvant Breast and Bowel Project B-18. J Clin Oncol 1997;15(7):2483-2493.

2. Wolmark N, Wang J, Mamounas E, Bryant $\mathrm{J}$, Fisher B. Preoperative chemotherapy in patients with operable breast cancer: nineyear results from National Surgical Adjuvant Breast and Bowel Project B-18. J Natl Cancer Inst Monogr 2001;(30):96-102.

3. Rastogi P, Anderson SJ, Bear HD, et al. Preoperative chemotherapy: updates of National Surgical Adjuvant Breast and Bowel Project Protocols B-18 and B-27. J Clin Oncol 2008;26(5):778-785.

4. Denley H, Pinder SE, Elston CW, Lee AH, Ellis IO. Preoperative assessment of prognostic factors in breast cancer. J Clin Pathol $2001 ; 54(1): 20-24$.

5. Rakha EA, El-Sayed ME, Green AR, et al. Biologic and clinical characteristics of breast 
cancer with single hormone receptor positive phenotype. J Clin Oncol 2007;25(30): 4772-4778.

6. Chuthapisith S, Eremin JM, Eremin O. Predicting response to neoadjuvant chemotherapy in breast cancer: molecular imaging, systemic biomarkers and the cancer metabolome (review). Oncol Rep 2008;20(4):699703.

7. Nicholson RI, Johnston SR. Endocrine therapy: current benefits and limitations. Breast Cancer Res Treat 2005;93(Suppl 1):S3-S10.

8. Tokunaga E, Oki E, Nishida $K$, et al. Trastuzumab and breast cancer: developments and current status. Int J Clin Oncol 2006;11(3):199-208.

9. Chagpar AB, Middleton LP, Sahin AA, et al. Accuracy of physical examination, ultrasonography, and mammography in predicting residual pathologic tumor size in patients treated with neoadjuvant chemotherapy. Ann Surg 2006;243(2):257-264.

10. Loo CE, Straver ME, Rodenhuis S, et al. Magnetic resonance imaging response monitoring of breast cancer during neoadjuvant chemotherapy: relevance of breast cancer subtype. J Clin Oncol 2011;29(6):660-666.

11. Miller BT, Abbott AM, Tuttle TM. The influence of preoperative MRI on breast cancer treatment. Ann Surg Oncol 2012;19(2):536540 .

12. Groheux D, Giacchetti S, Espié M, Rubello D, Moretti JL, Hindié E. Early monitoring of response to neoadjuvant chemotherapy in breast cancer with 18F-FDG PET/CT: defining a clinical aim. Eur J Nucl Med Mol Imaging 2011;38(3):419-425.

13. Tromberg BJ, Cerussi A, Shah N, et al. Imaging in breast cancer: diffuse optics in breast cancer-detecting tumors in premenopausal women and monitoring neoadjuvant chemotherapy. Breast Cancer Res 2005; 7(6):279-285.

14. Chance B, Nioka S, Zhang J, et al. Breast cancer detection based on incremental biochemical and physiological properties of breast cancers: a six-year, two-site study. Acad Radiol 2005;12(8):925-933.

15. Spinelli L, Torricelli A, Pifferi A, Taroni P, Danesini G, Cubeddu R. Characterization of female breast lesions from multi-wavelength time-resolved optical mammography. Phys Med Biol 2005;50(11):2489-2502.

16. Grosenick D, Moesta KT, Möller M, et al. Time-domain scanning optical mammography. I. Recording and assessment of mammograms of 154 patients. Phys Med Biol 2005;50(11):2429-2449.

17. Poplack SP, Tosteson TD, Wells WA, et al. Electromagnetic breast imaging: results of a pilot study in women with abnormal mammograms. Radiology 2007;243(2):350-359.

18. Zhu Q, Cronin EB, Currier AA, et al. Benign versus malignant breast masses: optical differentiation with US-guided optical imaging reconstruction. Radiology 2005;237(1):57-66.

19. Liang X, Zhang Q, Li C, Grobmyer SR, Fajardo LL, Jiang H. Phase-contrast diffuse optical tomography: pilot results in the breast. Acad Radiol 2008;15(7):859-866.

20. Choe R, Konecky SD, Corlu A, et al. Differentiation of benign and malignant breast tumors by in-vivo three-dimensional parallelplate diffuse optical tomography. J Biomed Opt 2009;14(2):024020.

21. Zhu Q, Hegde PU, Ricci A Jr, et al. Early-stage invasive breast cancers: potential role of optical tomography with US localization in assisting diagnosis. Radiology 2010;256(2):367-378.

22. Fang Q, Selb J, Carp SA, et al. Combined optical and X-ray tomosynthesis breast imaging. Radiology 2011;258(1):89-97.

23. Cerussi A, Hsiang D, Shah N, et al. Predicting response to breast cancer neoadjuvant chemotherapy using diffuse optical spectroscopy. Proc Natl Acad Sci U S A 2007;104(10):4014-4019.

24. Zhou C, Choe R, Shah N, et al. Diffuse optical monitoring of blood flow and oxygenation in human breast cancer during early stages of neoadjuvant chemotherapy. J Biomed Opt 2007;12(5):051903.

25. Zhu Q, Tannenbaum S, Hegde P, Kane M, Xu C, Kurtzman SH. Noninvasive monitoring of breast cancer during neoadjuvant chemotherapy using optical tomography with ultrasound localization. Neoplasia 2008;10(10):1028-1040.

26. Jiang S, Pogue BW, Carpenter CM, et al. Evaluation of breast tumor response to neo- adjuvant chemotherapy with tomographic diffuse optical spectroscopy: case studies of tumor region-of-interest changes. Radiology 2009;252(2):551-560.

27. Soliman H, Gunasekara A, Rycroft M, et al. Functional imaging using diffuse optical spectroscopy of neoadjuvant chemotherapy response in women with locally advanced breast cancer. Clin Cancer Res 2010;16(9):2605-2614.

28. Roblyer D, Ueda S, Cerussi A, et al. Optical imaging of breast cancer oxyhemoglobin flare correlates with neoadjuvant chemotherapy response one day after starting treatment. Proc Natl Acad Sci U S A 2011;108(35):14626-14631.

29. Zhu Q, Huang M, Chen N, et al. Ultrasound-guided optical tomographic imaging of malignant and benign breast lesions: initial clinical results of 19 cases. Neoplasia 2003;5(5):379-388

30. Zhu Q, Chen N, Kurtzman SH. Imaging tumor angiogenesis by use of combined near-infrared diffusive light and ultrasound. Opt Lett 2003;28(5):337-339.

31. Ogston KN, Miller ID, Payne S, et al. A new histological grading system to assess response of breast cancers to primary chemotherapy: prognostic significance and survival. Breast 2003;12(5):320-327.

32. Zhu Q, Xu C, Guo P, et al. Optimal probing of optical contrast of breast lesions of different size located at different depths by US localization. Technol Cancer Res Treat 2006;5(4):365-380.

33. Xu Y, Zhu Q. Imaging heterogeneous absorption distribution of advanced breast cancer by optical tomography. J Biomed Opt 2010;15(6):066007.

34. Biswal NC, Xu Y, Zhu Q. Imaging tumor oxyhemoglobin and deoxyhemoglobin concentrations with ultrasound-guided diffuse optical tomography. Technol Cancer Res Treat 2011;10(5):417-429.

35. Kuo WH, Chen CN, Hsieh FJ, et al. Vascularity change and tumor response to neoadjuvant chemotherapy for advanced breast cancer. Ultrasound Med Biol 2008;34(6):857-866. 